ఠ

\title{
Body, Brain, Life for Cognitive Decline (BBL-CD): protocol for a multidomain dementia risk reduction randomized controlled trial for subjective cognitive decline and mild cognitive impairment
}

This article was published in the following Dove Press journal:

Clinical Interventions in Aging

\author{
Mitchell McMaster' \\ Sarang Kim' \\ Linda Clare ${ }^{2}$ \\ Susan J Torres ${ }^{3}$ \\ Catherine D'Este ${ }^{4}$ \\ Kaarin J Anstey ${ }^{1,5,6}$
}

'Centre for Research on Ageing, Health and Wellbeing, Research

School of Population Health, The Australian National University, Canberra, ACT, Australia; ${ }^{2}$ Centre for Research in Ageing and Cognitive Health, University of Exeter, Exeter, UK; ${ }^{3}$ Institute for Physical Activity and Nutrition, School of Exercise and Nutrition Sciences, Deakin University, Geelong, VIC, Australia; ${ }^{4}$ National Centre for Epidemiology and Population Health, Research School of Population Health, The Australian National University, Canberra, ACT, Australia; ${ }^{5}$ Neuroscience Research Australia, Sydney, NSW, Australia; ${ }^{6}$ School of Psychology, University of New South Wales, Sydney, NSW, Australia
Correspondence: Mitchell McMaster Centre for Research on Ageing, Health and Wellbeing, Florey Building, 54 Mills Road, The Australian National University, Acton, Canberra, ACT 260I, Australia Tel +6I 2 6I25 635I

Email mitchell.mcmaster@anu.edu.au
Background: With no cure for dementia and the number of people living with the condition predicted to rapidly rise, there is an urgent need for dementia risk reduction and prevention interventions. Modifiable lifestyle risk factors have been identified as playing a major role in the development of dementia; hence, interventions addressing these risk factors represent a significant opportunity to reduce the number of people developing dementia. Relatively few interventions have been trialed in older participants with cognitive decline (secondary prevention).

Objectives: This study evaluates the efficacy and feasibility of a multidomain lifestyle risk reduction intervention for people with subjective cognitive decline (SCD) and mild cognitive impairment (MCI).

Methods: This study is an 8-week, two-arm, single-blind, randomized controlled trial (RCT) of a lifestyle modification program to reduce dementia risk. The active control group receives the following four online educational modules: dementia literacy and lifestyle risk, Mediterranean diet (MeDi), cognitive engagement and physical activity. The intervention group also completes the same educational modules but receives additional practical components including sessions with a dietitian, online brain training and sessions with an exercise physiologist to assist with lifestyle modification.

Results: Primary outcome measures are cognition (The Alzheimer's Disease Assessment Scale-Cognitive-Plus [ADAS-Cog-Plus]) and a composite lifestyle risk factor score for Alzheimer's disease (Australian National University - Alzheimer's Disease Risk Index [ANU-ADRI]). Secondary outcome measures are motivation to change lifestyle (Motivation to Change Lifestyle and Health Behaviour for Dementia Risk Reduction [MCLHB-DRR]) and health-related quality of life (36-item Short Form Health Survey [SF-36]). Feasibility will be determined through adherence to diet (Mediterranean Diet Adherence Screener [MEDAS] and Australian Recommended Food Score [ARFS]), cognitive engagement (BrainHQ-derived statistics) and physical activity interventions (physical activity calendars). Outcomes are measured at baseline, immediately post-intervention and at 3-and 6-month follow-up by researchers blind to group allocation.

Discussion: If successful and feasible, secondary prevention lifestyle interventions could provide a targeted, cost-effective way to reduce the number of people with cognitive decline going on to develop Alzheimer's disease (AD) and other dementias.

Keywords: dementia prevention, dementia risk reduction, secondary prevention, Alzheimer's disease, subjective cognitive decline, mild cognitive impairment, multidomain lifestyle intervention 


\section{Introduction}

The number of people with dementia is projected to rise to almost 75 million worldwide by 2030, and in the absence of a cure, there is an urgent need for strategies to reduce the number of people developing dementia. ${ }^{1}$ It has been estimated that up to half of all Alzheimer's disease (AD) cases worldwide may be attributed to seven modifiable risk factors, the majority of which reflect cardiovascular risks such as physical inactivity, hypertension, obesity, and diabetes. ${ }^{2}$

Primary prevention of dementia aims to reduce risk factors by focusing on improving the lifestyle of middle-aged people prior to or in the very earliest stages of the neuropathological changes which characterize $\mathrm{AD}$ and other types of dementia. ${ }^{3}$ An alternative strategy is secondary prevention, which aims to minimize any further damage or slow progression once symptoms of a disease begin to emerge. In the case of dementia, it is thought that the very earliest symptoms of disease are characterized by subjective cognitive decline (SCD) and later by mild cognitive impairment (MCI). ${ }^{3} \mathrm{SCD}$ is a condition in which people report cognitive deficits in day-to-day life, but these are not detectable with cognitive testing. ${ }^{4}$ The cognitive deficits of people with $\mathrm{MCI}$ are detectable with cognitive testing, but do not reach the threshold to meet the criteria for dementia. ${ }^{5}$ Both SCD and MCI are associated with increased risk of progressing to dementia, ${ }^{5,6}$ and the earliest stages of brain pathology found in dementia are also present in these conditions..$^{7-10}$

Although dementia is not considered to be a reversible condition, there are some indications that in these prodromal stages the brain may still retain sufficient neuroplasticity that the trajectory of the disease may be modifiable. For instance, in individuals with $\mathrm{MCI}$, conversion rates to $\mathrm{AD}$ are $7 \%$ at 1 year, $24 \%$ at 3 years and $59 \%$ at 6 years. ${ }^{11,12}$ Annually, approximately $25 \%$ of those with MCI revert back to normal cognitive status. ${ }^{13}$ There are differing explanations for this pattern of changes in cognitive status such as differing definitions for MCI, differences in testing procedures and test and retest effects that do not adequately represent these participants' true level of cognitive function. One explanation that cannot be discounted is that these low annual conversion rates and a high percentage of people reverting back to cognitively normal status suggest that this period may represent a "window of opportunity" for interventions to modify the disease course.

Three factors that have been identified by systematic reviews as having the potential to decrease lifestyle risk of dementia are diet, cognitive engagement, and physical activity. ${ }^{14,15}$

One dietary pattern that has shown promise in recent research is the Mediterranean diet (MeDi). The MeDi is a dietary pattern which is predominantly plant-based, with a high intake of vegetables, fruits, nuts and legumes, moderately high intake of fish, low intake of red meat, and includes extra virgin olive oil as the main source of fat. ${ }^{16}$ The MeDi has been shown to decrease dementia risk indirectly through altering cardiovascular risk factors, ${ }^{17}$ as well as directly through lower levels of neuropathology such as amyloid plaques, ${ }^{18}$ brain atrophy, ${ }^{19}$ and structural connectivity. ${ }^{20}$

One of the most compelling studies in the area of cognitive engagement is the Advanced Cognitive Training in Vital Elderly (ACTIVE) trial. ${ }^{21}$ The ACTIVE trial was a computerized cognitive training randomized controlled trial (RCT), comparing memory, reasoning, and speed of processing training conditions to a control condition. The speed of processing training group showed higher cognition and lower incident dementia at 10 years post-intervention, relative to the control group. ${ }^{21,22}$ Although the ACTIVE trial was conducted with a cognitively normal sample over the age of 65 years, these effects have yet to be replicated in a group with cognitive decline, such as SCD or MCI.

Similar to diet, physical activity is both indirectly and directly related to dementia risk. Physical activity has repeatedly been shown to be protective against cardiovascular risk factors for dementia ${ }^{23}$ and to reduce $\mathrm{AD}$ risk directly through a host of neuronal mechanisms, including downregulating pathways that lead to amyloid and tau production. ${ }^{24}$ In a review of modifiable risk factors, physical inactivity has the highest attributable risk of the seven dementia risk factors identified ( $18 \%$ of all dementia cases in Australia). ${ }^{25}$ Although the bulk of evidence for these factors comes from primary prevention studies (eg, with middle-aged adults), systematic reviews have highlighted the need to explore such approaches as secondary prevention interventions in people experiencing the earliest stages of cognitive decline. ${ }^{26-28}$

Some early studies in the area of secondary prevention have shown encouraging results. A 12-week, single-arm intervention for community-dwelling people with MCI $(\mathrm{n}=127$, average age 70.7 years) involving MeDi, omega-3 supplements, physical activity, cognitive stimulation, neurofeedback, and meditation achieved positive results. ${ }^{29}$ At the final follow-up, $84 \%$ of the participants showed statistically significant improvements in cognition and $53 \%$ of a subsample $(n=17)$ that underwent neuroimaging showed hippocampal growth. Limitations of this study were that there was no control group and participants were not randomized, making it difficult to determine whether the effects were due to the intervention, and the low number of participants in the neuroimaging subsample means that these changes may not be representative of the whole sample. The study concluded 
that further multidomain RCTs should be conducted in participants experiencing cognitive decline. One multidomain RCT was conducted in community-dwelling frail and prefrail participants over the age of 65 years, which compared interventions for physical activity, cognitive activity, nutrition, and a combination of the three interventions against a control group. ${ }^{30}$ Over 12 months, improvements in different domains of cognition were seen with all groups except the physical activity intervention group, in comparison to declines seen in the control group. As one of the limitations, the study noted that as physical frailty was the primary target of the intervention, only $7 \%$ of the sample had a Mini Mental State Examination (MMSE) score less than 26 and the study may have been underpowered to detect all cognitive effects. The study recommended that further multidomain RCTs should be trialed in participants with greater levels of cognitive impairment as greater benefits may be possible with this population.

Although preliminary studies on secondary prevention do report positive outcomes, there is a need for more rigorous, multidomain studies such as RCTs, designed specifically to look at relevant outcomes such as cognition and lifestyle risk factors.

\section{Objectives}

The Body, Brain, Life (BBL) interventions are a suite of multidomain, primary dementia risk reduction interventions. ${ }^{31-33}$ The original intervention included educational modules only and more recently face-to-face physical activity and dietary components have been added. ${ }^{32}$ The present study, Body, Brain, Life for Cognitive Decline (BBL-CD), draws from the earlier interventions but introduces some new components and adaptations so that it is suitable for participants with cognitive impairment. This study evaluates the feasibility and efficacy of adapting this program for a cognitively impaired population.

The specific aims of the study are to

1. evaluate the efficacy of BBL-CD in the prevention of further cognitive decline;

2. evaluate the efficacy of BBL-CD to reduce overall lifestyle risk of $\mathrm{AD}$ and other dementias and

3. evaluate the feasibility of BBL-CD through tracking intervention adherence.

\section{Methods}

\section{Study design}

The study is an 8-week, two-arm, parallel group RCT. The intervention focuses on the following three domains of lifestyle: diet, cognitive engagement, and physical activity.
The active control group will undertake four online educational modules and the intervention group will undertake the same online modules complemented by practical and faceto-face sessions with interventionists. The study is expected to complete data collection in late 2018. This study is registered with the Australian and New Zealand Clinical Trials Registry (ACTRN12617000792325) and ethical approval for conducting this study was granted by the Australian National University Human Research Ethics Committee (Protocol: 2016/360). The study has been planned and conducted in accordance with the revised Declaration of Helsinki, ${ }^{34}$ and all participants provided written informed consent to participate in this study. This protocol was written to conform with the Standard Protocol Items: Recommendations for Interventional Trials (SPIRIT) checklist. ${ }^{35}$

\section{Participants}

Participants are community-dwelling individuals with MCI or SCD recruited through advertisements in community newsletters, local print media, and radio. The inclusion criteria are as follows: living in the Australian Capital Territory or Queanbeyan, New South Wales; aged 65 years or over; owning a computer with Internet access; having sufficient English language skills; being prepared to make lifestyle changes to improve health; and having a medical diagnosis of MCI or meeting the Jessen criteria ${ }^{4}$ for SCD (clinically normal on objective assessment, self/informant-reported cognitive decline, decline not better accounted for by major medical and neurological or psychiatric diagnosis). The criteria for MCI are met if the participant has previously received a diagnosis of MCI from a suitably qualified medical professional such as a neuropsychologist or geriatrician (no exact criteria for MCI diagnosis are specified). The criteria for SCD are met if the participant expresses the view that they have experienced a decline in any domains of cognitive function in the past 5 years.

Exclusion criteria are as follows: currently participating in any lifestyle change interventions; have a diagnosis of $\mathrm{AD}$ or another form of dementia and have major psychiatric, neurological, or physical problems which would prevent them from taking part in a lifestyle change program.

All inclusion and exclusion criteria are assessed via an initial phone call to the research team when potential participants express interest in participating in the study. Participants meeting the criteria are sent an information sheet about the study and a consent form to sign and return.

If at any of the testing points cognitive testing is indicative of potential AD (The Alzheimer's Disease Assessment ScaleCognitive Subscale [ADAS-Cog] >12), participants are 
referred to their general practitioner (GP) for cognitive testing. If found to have probable $\mathrm{AD}$, the participant is withdrawn and no further data are collected; these participants are still allowed to continue participating in the practical components of the intervention, if they choose to (ie, participants will not be penalized or disadvantaged by a dementia diagnosis).

\section{Randomization and stratification}

After completing baseline data collection, participants are randomized in a 1:1 ratio, within strata defined by gender, baseline ADAS-Cog-Plus (above or below the median value of 7.0) and baseline ANU-ADRI (above or below the median value of 10.0), to intervention or control groups in permuted blocks of eight. The randomization sequence is generated from www.sealedenvelope.com by an independent researcher $(\mathrm{RB})$.

\section{Interventions}

\section{Active control}

The active control group undertakes an 8-week, four-module, online educational course on dementia risk reduction and effective goal setting. The four modules are as follows:

1. Dementia literacy and lifestyle risk for AD (week 1): this module describes SCD, MCI, AD, and dementia, modifiable and non-modifiable risk factors and effective goal setting and rewards.

2. Diet (week 2): the diet module explains the importance of a healthy diet in maintaining a healthy brain. It explains the general principles of the MeDi and the scientific evidence that supports MeDi as a diet associated with lower levels of chronic disease and dementia.

3. Cognitive engagement (week 4): this module reviews the evidence for a cognitively engaged lifestyle being related to lower levels of dementia, different forms of cognitive engagement and how to increase cognitive engagement in everyday life.

4. Physical activity (week 6): this module discusses the evidence for an adequate level of physical activity in risk reduction of chronic disease and dementia. It also covers the importance of engaging in a combination of aerobic, strength, balance, and flexibility exercises and the Australian Department of Health's Physical Activity and Sedentary Behaviour Guidelines for people $\geq 65$ years. $^{36}$

The modules are interactive, including some questions to check participants' understanding of the content, and give the opportunity to provide information about aspects of their lifestyle and ways in which they might be able to modify their behavior. Each module provides examples of how to set specific, measurable, achievable, relevant, and timed (SMART) goals for the particular domain. Each module takes approximately 1-2 hours to complete and can be completed across multiple sittings, if desired.

\section{Intervention}

The intervention group receives the same educational modules as the active control group, but each module is complemented by practical components to assist with the implementation of changes into the lifestyle in a sustainable way. The practical components are as follows:

1. Diet: to reinforce the content of the diet module, the participants have three face-to-face sessions with a dietitian, including an initial 1-hour session (week 3 ) and two further 30-minute follow-up sessions (weeks 10 and 21). In these sessions, the dietitian reviews the participant's previous diet assessment results, discusses any barriers to adherence they are experiencing and ways in which the participant could achieve greater adherence to the MeDi. The MeDi intervention is adapted from the study by Estruch et al. ${ }^{37}$ Recommendations are as follows: 1 ) $\geq 5$ servings of vegetables/day, including two servings of raw vegetables; 2 ) $\geq 3$ servings of fruit/day; 3 ) $\geq 3$ serves of fish or seafood/ week, including one serving of fatty fish; 4) $\geq 3$ servings of legumes/week; 5) $\geq 3$ servings of nuts/week; 6) preferentially consume white meat, instead of red meat; 7) $<1$ serving of red meat/day; 8) using olive oil as the main oil for cooking and dressing; 9) $\geq 4$ servings of olive oil/day; 10) $<1$ serving of butter, margarine, or cream/ day; 11) $\geq 7$ servings of wine/week; 12) $\geq 2$ servings of sofrito sauce/week (tomato, garlic, onion, and olive oil); 13) $<1$ serving of sweet or carbonated beverages/day; and 14) $<3$ servings of commercial sweets or pastries/week.

2. Cognitive engagement: to enable the participants to live a more cognitively engaged lifestyle, they are provided with a BrainHQ ${ }^{38}$ account (week 5) and asked to participate in two executive functions and two memory tasks for 30 minutes each (total 2 hours) per week. The four tasks are as follows: Double Decision (divided and selective attention, speed of processing, dual task, and useful field of view); Freeze Frame (visual phasic and tonic attention, inhibitory control and motor response inhibition); Syllable Stacks (auditory working memory); and Memory Grid (auditory spatial memory). The exercises are psychophysically adaptive and the parameters within each stimulus set are adjusted for an individual participant to maintain $\sim 80 \%$ criterion accuracy by increasing or 
decreasing task difficulty systematically with correct/ incorrect responses.

3. Physical activity: participants attend an initial 45-minute session (week 7) and two 30-minute follow-up sessions with an exercise physiologist (weeks 10 and 21). Based on the participant's medical conditions, current level of exercise and physical activity preferences, a weekly exercise regime is developed with the eventual aim to increase physical activity level to 150 minutes of moderate exercise per week. If participants are already undertaking this level of exercise or greater, the goal is maintenance and combining different forms of exercises (eg, aerobic, strength, balance, and flexibility exercises). In the follow-up sessions, the exercise physiologist discusses progress, any barriers that the participant is experiencing and any modifications to the exercise regime, and (if suitable) increases toward 150 minutes total exercise duration. To be involved in these sessions, participants must have a medical clearance form signed by a GP, detailing any medical conditions or medications which may impact the participant's ability to undertake exercise and approving the participant to undertake moderate physical exercise. A week-by-week summary of the intervention is shown in Figure 1.

\section{Modifications made to previous BBL interventions}

The BBL intervention has been modified each time it has been conducted, based on participant feedback on the previous version. ${ }^{31-33}$ Further modifications were made for this study to increase the suitability for an older population experiencing cognitive decline. The overall size and amount of information that participants are required to learn and remember in the online modules were decreased based on feedback from middle-aged participants that there was too much information to read and remember. Previous versions of BBL contained seven or eight informational modules on different dementia risk factors, followed by 4 weeks of revision. To enable a population experiencing cognitive deficits to effectively learn and modify their behavior without being overwhelmed, the content was reduced to three risk factors that could be most easily targeted to bring about effective risk reductions. Previous BBL studies involved one module on a new risk factor per week for the first 8 weeks. In BBL-CD, after a module on a risk factor is introduced, a week without a module is allotted for participants to implement these changes into their lifestyle. In BBL-CD, in week 8 at the conclusion of all the modules the participants have a week for revision and are sent a one-page summary of each module to convey the key messages of the intervention. Each module summary also provides an example of a SMART goal for that specific risk factor, so that participants can continue to modify their behavior beyond the initial 8-week intervention period. Specific modifications to the modules included the following: the diet module and practical intervention were modified from the previous BBL focusing on a healthy balanced diet to the MeDi in consultation with study dietitians; the brain training is a novel inclusion due to a change in the participant group to one experiencing cognitive decline; and the physical activity program is now focused on moderate physical activity, rather than a structured walking program based on feedback from participants who felt that the walking program was too restrictive. These modified modules and practical components were piloted with a small group of individuals $(n=7)$ who volunteered to be participants but failed to meet a small number of the selection criteria (eg, less than 65 years of age, medical condition preventing dietary modification, etc). Feedback on each module and practical component was collected, and although overall feedback was positive, some modifications were implemented, eg, module wording changes and materials used during practical components.

Due to the high attrition rate in longitudinal intervention studies, several strategies to decrease attrition are being implemented. To make participants feel included and valued in the scientific process, newsletters are periodically sent about the progress of the study; participants are informed about any publications/conference presentations from the research; and participants are thanked after attending data collection and intervention sessions. The intervention group is termed the "Lifestyle Intervention Group" and the control group is termed the "Online Education Group", such that participants in the control group do not feel they are receiving an intervention that is unlikely to have any effect.

\section{Outcome measures}

\section{Primary outcome measures}

Cognition

Cognition is measured by the ADAS-Cog-Plus, ${ }^{39}$ which contains the standard ADAS-Cog items (word recall, naming objects and fingers, following commands, constructional praxis, ideational praxis, orientation, word recognition, language production, language comprehension, and word finding difficulty $)^{40}$ with additional measures for executive function (trail making task, ${ }^{41}$ symbol digit modalities test, ${ }^{42}$ category fluency task, $)^{43}$ and instrumental activities of daily living (Pfeffer Functional Activities Questionnaire items 


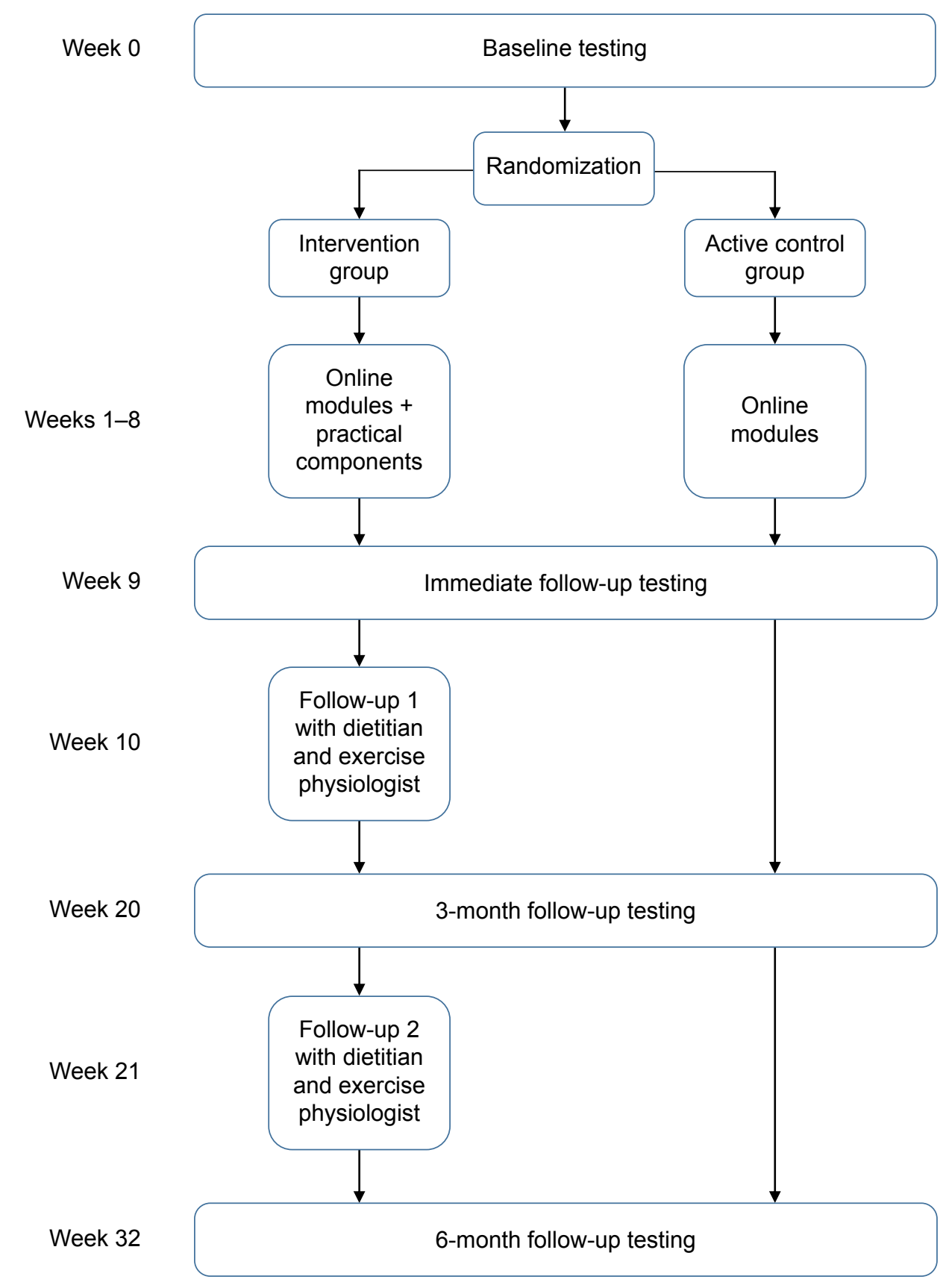

Figure I Timing of intervention components and testing periods.

1, 2, 4, 7 and 9). ${ }^{44}$ The inclusion of executive function and instrumental activities of daily living items is designed to maximize sensitivity to early-stage deficits as seen in SCD and MCI. The ADAS-Cog is scored from 0 to 70 , with higher scores indicating greater levels of cognitive impairment. ADAS-Cog has good reliability (Cronbach's $\alpha=0.83$, testretest $=0.93)^{45}$ and has been shown to discriminate between individuals with normal cognition, $\mathrm{MCI}$ and $\mathrm{AD} .{ }^{40}$ The inclusion of a full battery of cognitive measures is a new inclusion for the BBL studies, given a population experiencing cognitive decline.

\section{Lifestyle risk of $A D$}

Lifestyle risk factors for $\mathrm{AD}$ are measured by the Australian National University - Alzheimer's Disease Risk Index (ANU-ADRI) ${ }^{46}$ The ANU-ADRI covers 11 AD risk factors (eg, age, education and smoking) and four lifestyle factors that are protective against $\mathrm{AD}$ (eg, physical activity, fish intake, and cognitive activity). The ANU-ADRI applies an $\mathrm{AD}$ risk score for the level of each risk or protective factor, based on ORs derived from systematic reviews. ${ }^{46}$ The ANUADRI yields a score ranging from -14 (highly protective lifestyle) to 73 (high-risk lifestyle). The ANU-ADRI has 
been validated against three large, international longitudinal cohort studies and was shown to be a valid predictor of development of AD. ${ }^{47}$

\section{Secondary outcome measures \\ Motivation}

Motivation to change lifestyle is being assessed by the Motivation to Change Lifestyle and Health Behaviour for Dementia Risk Reduction (MCLHB-DRR). ${ }^{48}$ This is a 27-item scale, which was developed based on the principles of the Health Belief Model. It was developed and validated specifically to look at motivation to change lifestyle in health and dementia lifestyle interventions.

\section{Health-related quality of life}

The 36-item Short Form Health Survey (SF-36) ${ }^{49}$ is being used to assess general health-related quality of life. The SF-36 has 36-items which form an eight-scale profile of health-related quality of life which can be summarized into a physical component summary (PCS) and mental component summary (MCS). An overall score of 0-100 can be calculated from the mean values of the eight scales. Higher scores indicate better health-related quality of life. The SF-36 is commonly used in health research, especially in the evaluation of interventions, as it is easily converted to quality of life years (QALYs), which gives a quantitative measure of the benefit of an intervention and can be used in a cost-benefit analysis. ${ }^{50}$

\section{Anthropometric measures}

Height, weight, waist, and hip circumference are collected. Height to the nearest millimeter is measured using a stadiometer (Seca, Hamburg, Germany), and weight to the nearest $0.1 \mathrm{~kg}$ is measured with digital scales (Propert, Sydney, NSW, Australia). ${ }^{51}$ Body mass index is calculated as weight $(\mathrm{kg})$ divided by height squared $\left(\mathrm{m}^{2}\right)$. Waist circumference is measured to the nearest centimeter midway between the lowest rib margin and the iliac crest. ${ }^{51}$ Hip circumference is measured to the nearest centimeter at the point yielding the maximum circumference over the buttocks. ${ }^{51}$

\section{Feasibility and adherence measures}

The feasibility of the lifestyle intervention for this participant group is measured through adherence to diet, cognitive engagement, and physical activity. Additional qualitative interviews with a subsample of participants will be undertaken at the conclusion of the study to investigate other factors affecting feasibility and adherence to interventions such as this.

\section{Dietary assessment}

Dietary adherence to the MeDi is assessed using a validated 14-point Mediterranean Diet Adherence Screener (MEDAS).$^{52}$ A score of 0 or 1 is assigned to each item, with a maximum score of 14 indicating greatest adherence to the MeDi. The MEDAS has good convergent validity with a 137-item MeDi food frequency questionnaire and shows significant associations with health indices such as fasting glucose, total:high-density lipoprotein (HDL) cholesterol ratio, triglycerides and coronary artery disease risk. ${ }^{52}$

Dietary quality is assessed with a food-based diet quality index, the Australian Recommended Food Score (ARFS). ${ }^{53}$ The ARFS is aligned with Australian Dietary Guidelines ${ }^{54}$ and the Australian Guide to Healthy Eating ${ }^{55}$ recommendations. The ARFS total ranges from 0 to 73 and includes eight subscales: vegetables (0-21), fruit (0-12), protein (0-7), vegetarian protein alternatives $(0-6)$, grains $(0-13)$, dairy $(0-11)$, water $(0-1)$, and sauces and condiments (0-2). Higher scores indicate greater compliance with the Australian Dietary Guidelines and therefore better diet quality. The ARFS has demonstrated good validity and reproducibility. ${ }^{53}$

\section{Cognitive engagement}

Duration of engagement with cognitive training and levels completed are tracked automatically via the BrainHQ website for the intervention group.

\section{Physical activity}

Participants track their daily physical activity on a paperbased physical activity calendar. The activity, intensity (on a 20-point scale), ${ }^{56}$ and duration of the physical activity are recorded. Participants are asked to start completing this measure from the day of baseline testing onward and return these to the research team by electronic scan and email or by post at the end of every month.

\section{Data collection}

There are four primary data collection points in the study as follows: baseline (week 0); immediate post-intervention testing (week 9); 3-month testing (week 20); and 6-month testing (week 32). Research staff collecting data are blind to group allocation. Not all outcome measures are administered at each time point, and a summary of this is presented in Table 1.

\section{Statistical methods}

\section{Sample size calculation}

The required sample size was determined to enable detection of a difference between groups at 6 months of 0.70 SDs for the primary outcomes. This equates to approximately 
Table I Summary of outcomes measured at data collection points

\begin{tabular}{|l|c|c|c|c|}
\hline & $\begin{array}{c}\text { Baseline } \\
\text { (week 0) }\end{array}$ & $\begin{array}{c}\text { Immediate post- } \\
\text { intervention (week 9) }\end{array}$ & $\begin{array}{c}\text { 3-month follow-up } \\
\text { (week 20) }\end{array}$ & $\begin{array}{c}\text { 6-month follow-up } \\
\text { (week 32) }\end{array}$ \\
\hline Primary outcomes & & & $\checkmark$ & $\checkmark$ \\
\hline Cognition & $\checkmark$ & $\checkmark$ & $\checkmark$ & $\checkmark$ \\
\hline Lifestyle risk & $\checkmark$ & & $\checkmark$ & $\checkmark$ \\
\hline Secondary outcomes & $\checkmark$ & $\checkmark$ & $\checkmark$ & $\checkmark$ \\
\hline Motivation & $\checkmark$ & $\checkmark$ & $\checkmark$ & $\checkmark$ \\
\hline Health & $\checkmark$ & & $\checkmark$ & $\checkmark$ \\
\hline Anthropometry & $\checkmark$ & $\checkmark$ & $\checkmark$ \\
\hline Feasibility outcomes & $\checkmark$ & $\checkmark$ & \\
\hline Mediterranean diet & $\checkmark$ & & \\
\hline Dietary quality & \multicolumn{2}{|c|}{$\checkmark$} & & $\checkmark$ \\
\hline Cognitive engagement & Collected daily from week 5 onward for the intervention group & \\
\hline Physical activity & Collected daily from baseline (week 0) onward & & $\checkmark$ \\
\hline
\end{tabular}

Note: Data collection at baseline, 3-month and 6-month follow-up is conducted face-to-face, and immediate post-intervention assessments are online questionnaires.

4.2 units for the ADAS-Cog-Plus and 4.0 units on the ANUADRI, both of which are clinically significant magnitudes. This requires a minimum of 72 participants ( 36 participants per arm) at the final follow-up period. Accounting for a potential attrition rate of $10 \%$ over four testing periods (60\% remaining) yields a target sample size at baseline of 60 participants per group (120 in total).

\section{Planned analyses}

Linear mixed modeling to compare outcomes between intervention and control groups at each follow-up time, adjusted for baseline values of the outcome, will be conducted using complete cases as the primary analysis and full intentionto-treat analysis using multiple imputation to account for missing data as sensitivity analysis.

\section{Discussion}

There is a clear need for dementia prevention and risk reduction studies, given the anticipated rise in the number of people with dementia in the coming years. ${ }^{1}$ Several authors have argued that secondary prevention (older populations showing some symptoms of cognitive decline) is an avenue that warrants further exploration. ${ }^{26-28}$ Drawing on epidemiological findings and previous trials and applying this to an older, high-risk group is the strategy that has been adopted for this study. Secondary prevention, if feasible and effective, provides a targeted approach to reducing the number of people developing dementia. Such intervention programs could be implemented through primary care providers who identify individuals in the high-risk SCD/MCI groups. Interventions could be run as collaborations between medical and allied health professionals and could be tailored to individuals' specific needs based on their risk factor profile. Targeting a small number of people at very high risk of developing dementia would likely prove to be more cost-effective than targeting a larger number of people at lower risk.

The first step to showing the value of secondary prevention is to conduct randomized controlled studies to demonstrate feasibility and positive effects, such as reductions in risk factors or improvements in cognition in the SCD/MCI group. As a smaller, proof of concept study this research focuses on three of the most important risk factors: diet, cognitive engagement and physical exercise. This study aims to evaluate the efficacy of BBL-CD to prevent further cognitive decline and dementia risk profile and to evaluate the feasibility of the intervention for this participant group. These findings would provide proof of concept for a larger, longer secondary prevention trial with this group in the future.

\section{Strengths and limitations}

A major strength of this project is that all the components of the intervention have previously been used in separate successful interventions, ${ }^{21,31,32,37}$ but not with a group experiencing cognitive decline. The efficacy and feasibility of dementia risk reduction interventions in this group have been identified as a major knowledge gap by systematic reviews, ${ }^{26-28}$ and the use of multidomain interventions to combat multiple risk factors simultaneously is considered to be the "gold standard" for risk reduction and prevention interventions. ${ }^{28}$

The greatest limitation of this research is the short follow-up time. Systematic reviews of RCTs in dementia 
risk reduction interventions recommend follow-up times of a year or longer. ${ }^{27}$ Due to the paucity of research in the area of secondary prevention, it is important to first establish the feasibility of an adaptation of the BBL intervention to the SCD/MCI group and test the hypotheses that these individuals do appear to retain sufficient neuroplasticity to warrant a larger and longer trial.

\section{Data sharing statement}

Deidentified individual data sets are available indefinitely upon request to the authors following publication of the results of the study.

\section{Acknowledgments}

We thank Dr Richard Burns for his advice and assistance with the randomization of the sample. MM's PhD Scholarship is supported by Dementia Australia Research Foundation, the Australian National University and Dementia Collaborative Research Centre. Additional research funding is provided by the NHMRC Centre for Research Excellence in Cognitive Health, the Australian National University; Neuroscience Research Australia, University of New South Wales; the 2017 Royal Commonwealth Society Phyllis Montgomery Award; NHMRC Fellowship Funds (KA: APP1102694); and the Dementia Collaborative Research Centre (funds for precursor study). SK and LC are funded by the NHMRC Centre for Research Excellence in Cognitive Health. None of the funding bodies played any part in the study conception, design, or conduct of the study.

\section{Disclosure}

The authors report no conflicts of interest in this work.

\section{References}

1. Alzheimer's Disease International. World Alzheimer's Report 2015. The Global Impact of Dementia. An Analysis of Prevalence, Incidence, Cost and Trends. 2015.

2. Barnes DE, Yaffe K. The projected effect of risk factor reduction on Alzheimer's disease prevalence. Lancet Neurol. 2011;10(9):819-828.

3. Fratiglioni L, Winblad B, von Strauss E. Prevention of Alzheimer's disease and dementia. Major findings from the Kungsholmen Project. Physiol Behav. 2007;92(1-2):98-104.

4. Jessen F, Amariglio RE, van Boxtel M, et al. A conceptual framework for research on subjective cognitive decline in preclinical Alzheimer's disease. Alzheimers Dement. 2014;10(6):844-852.

5. Petersen RC. Mild cognitive impairment as a diagnostic entity. J Intern Med. 2004;256(3):183-194.

6. Mitchell AJ, Beaumont H, Ferguson D, Yadegarfar M, Stubbs B. Risk of dementia and mild cognitive impairment in older people with subjective memory complaints: meta-analysis. Acta Psychiatr Scand. 2014; 130(6):439-451.

7. Amariglio RE, Becker JA, Carmasin J, et al. Subjective cognitive complaints and amyloid burden in cognitively normal older individuals. Neuropsychologia. 2012;50(12):2880-2886.
8. Pike KE, Savage G, Villemagne VL, et al. Beta-amyloid imaging and memory in non-demented individuals: evidence for preclinical Alzheimer's disease. Brain. 2007;130(Pt 11):2837-2844.

9. Shi F, Liu B, Zhou Y, Yu C, Jiang T. Hippocampal volume and asymmetry in mild cognitive impairment and Alzheimer's disease: metaanalyses of MRI studies. Hippocampus. 2009;19(11):1055-1064.

10. Perrotin A, de Flores R, Lamberton F, et al. Hippocampal subfield volumetry and $3 \mathrm{D}$ surface mapping in subjective cognitive decline. J Alzheimers Dis. 2015;48(s1):S141-S150.

11. Bradfield NI, Ellis KA, Savage G, et al. Baseline amnestic severity predicts progression from amnestic mild cognitive impairment to Alzheimer disease dementia at 3 years. Alzheimer Dis Assoc Disord. 2018;32(3):190-196.

12. Wallin A, Nordlund A, Jonsson M, et al. The Gothenburg MCI study: design and distribution of Alzheimer's disease and subcortical vascular disease diagnoses from baseline to 6-year follow-up. J Cereb Blood Flow Metab. 2016;36(1):114-131.

13. Malek-Ahmadi M. Reversion from mild cognitive impairment to normal cognition: a meta-analysis. Alzheimer Dis Assoc Disord. 2016; 30(4):324-330.

14. Xu W, Tan L, Wang HF, et al. Meta-analysis of modifiable risk factors for Alzheimer's disease. J Neurol Neurosurg Psychiatry. 2015;86(12): 1299-1306.

15. Baumgart M, Snyder HM, Carrillo MC, Fazio S, Kim H, Johns H. Summary of the evidence on modifiable risk factors for cognitive decline and dementia: a population-based perspective. Alzheimers Dement. 2015;11(6):718-726.

16. Davis C, Bryan J, Hodgson J, Murphy K. Definition of the Mediterranean diet; a literature review. Nutrients. 2015;7(11):9139-9153.

17. Salas-Salvadó J, Becerra-Tomás N, García-Gavilán JF, Bulló M, Barrubés L. Mediterranean diet and cardiovascular disease prevention: what do we know? Prog Cardiovasc Dis. 2018;61(1):62-67.

18. Berti V, Walters M, Sterling J, et al. Mediterranean diet and 3-year Alzheimer brain biomarker changes in middle-aged adults. Neurology. 2018;90(20):e1789-e1798.

19. Luciano M, Corley J, Cox SR, et al. Mediterranean-type diet and brain structural change from 73 to 76 years in a Scottish cohort. Neurology. 2017;88(5):449-455.

20. Pelletier A, Barul C, Féart C, et al. Mediterranean diet and preserved brain structural connectivity in older subjects. Alzheimers Dement. 2015;11(9):1023-1031.

21. Rebok GW, Ball K, Guey LT. Ten-year effects of the ACTIVE cognitive training trial on cognition and everyday functioning in older adults. $J$ Am Geriatr Soc. 2014;62(1):16.

22. Edwards JD, Xu H, Clark D, Ross LA, Unverzagt FW. The ACTIVE study: what we have learned and what is next? Cognitive training reduces incident dementia across ten years. Alzheimer's Dementia. 2016; 12(7):P212.

23. Lanier JB, Bury DC, Richardson SW. Diet and Physical activity for cardiovascular disease prevention. Am Fam Physician. 2016;93(11): 919-924.

24. Loprinzi PD, Frith E, Ponce P. Memorcise and Alzheimer's disease. Phys Sportsmed. 2018;46(2):145-154.

25. Ashby-Mitchell K, Burns R, Shaw J, Anstey KJ. Proportion of dementia in Australia explained by common modifiable risk factors. Alzheimers Res Ther. 2017;9(1):11.

26. Horr T, Messinger-Rapport B, Pillai JA. Systematic review of strengths and limitations of randomized controlled trials for non-pharmacological interventions in mild cognitive impairment: focus on Alzheimer's disease. J Nutr Health Aging. 2015;19(2):141-153.

27. Smart CM, Karr JE, Areshenkoff CN, et al. Non-pharmacologic interventions for older adults with subjective cognitive decline: systematic review, meta-analysis, and preliminary recommendations. Neuropsychol Rev. 2017;27(3):245-257.

28. Williams JW, Plassman BL, Burke J, Holsinger T, Benjamin S. Prevention Alzheimer's Disease and Cognitive Decline. Rockville, MD: Agency for Healthcare Research and Quality (US); 2010. 
29. Fotuhi M, Lubinski B, Trullinger M, et al. A Personalized 12-week "Brain Fitness Program" for improving cognitive function and increasing the volume of hippocampus in elderly with mild cognitive impairment. J Prev Alzheimers Dis. 2016;3(3):133-137.

30. Ng TP, Ling A, Feng L, et al. Cognitive effects of multi-domain interventions among pre-frail and frail community-living older persons: randomized controlled trial. J Gerontol Series A. 2017.

31. Anstey KJ, Bahar-Fuchs A, Herath P, et al. Body brain life: a randomized controlled trial of an online dementia risk reduction intervention in middle-aged adults at risk of Alzheimer's disease. Alzheimers Dement. 2015;1(1):72-80.

32. Kim S, Mcmaster M, Torres S, et al. Protocol for a pragmatic randomised controlled trial of Body Brain Life-General Practice and a Lifestyle Modification Programme to decrease dementia risk exposure in a primary care setting. BMJ Open. 2018;8(3):e019329.

33. Anstey KJ, Kim S [homepage on the Internet]. The Body, Brain, LifeFit (BBL-FIT) Program - A Pilot Study to Evaluate the Feasibility of the BBL-FIT Online Lifestyle Program in Middle-Aged Adults at Risk of Dementia (ACTRN12615000822583). Australian and New Zealand Clinical Trial Registry. Available from: https:/www.anzctr. org.au/Trial/Registration/TrialReview.aspx?id=368897;2015. Accessed October 24, 2018.

34. World Medical Association. World Medical Association Declaration of Helsinki: ethical principles for medical research involving human subjects. JAMA. 2013;310(20):2191.

35. Chan AW, Tetzlaff JM, Altman DG, et al. SPIRIT 2013 statement: defining standard protocol items for clinical trials. Ann Intern Med. 2013;158(3):200-207.

36. Australian Department of Health [homepage on the Internet]. Physical Activity and Sendentary Behaviour Guidelines. Physical Activity and Sendentary Behaviour Guidelines. Available from: http://www.health. gov.au/internet/main/publishing.nsf/content/health-pubhlth-strategphys-act-guidelines\#chba. Accessed 1/5/2018.

37. Estruch R, Ros E, Salas-Salvadó J, et al. Primary prevention of cardiovascular disease with a Mediterranean diet. $N$ Engl J Med. 2013; 368(14):1279-1290.

38. Posit Sceince [homepage on the Internet]. BrainHQ. Available from: http://www.brainhq.com. Accessed October 3, 2018.

39. Skinner J, Carvalho JO, Potter GG, et al. The Alzheimer's Disease Assessment Scale-Cognitive-Plus (ADAS-Cog-Plus): an expansion of the ADAS-Cog to improve responsiveness in MCI. Brain Imaging Behav. 2012;6(4):489-501.

40. Kueper JK, Speechley M, Montero-Odasso M. The Alzheimer's Disease Assessment Scale-Cognitive Subscale (ADAS-Cog): modifications and responsiveness in pre-dementia populations. A narrative review. J Alzheimers Dis. 2018;63(2):423-444.
41. Reitan RM. Trail Making Test: Manual for Administration and Scoring. Tucson, AZ: Reitan Neuropsychology Laboratory; 1992.

42. Smith A. Symbol Digit Modalities Test. Los Angeles, CA: Western Psychological Services; 1982.

43. Lucas JA, Ivnik RJ, Smith GE, et al. Mayo's older Americans normative studies: category fluency norms. J Clin Exp Neuropsychol. 1998; 20(2):194-200.

44. Pfeffer RI, Kurosaki TT, Harrah CH, Chance JM, Filos S. Measurement of functional activities in older adults in the community. J Gerontol. 1982;37(3):323-329.

45. Cano SJ, Posner HB, Moline ML, et al. The ADAS-cog in Alzheimer's disease clinical trials: psychometric evaluation of the sum and its parts. J Neurol Neurosurg Psychiatry. 2010;81(12):1363-1368.

46. Anstey KJ, Cherbuin N, Herath PM. Development of a new method for assessing global risk of Alzheimer's disease for use in population health approaches to prevention. Prev Sci. 2013;14(4):411-421.

47. Anstey KJ, Cherbuin N, Herath PM, et al. A self-report risk index to predict occurrence of dementia in three independent cohorts of older adults: the ANU-ADRI. PLoS One. 2014;9(1):e86141.

48. Kim S, Sargent-Cox K, Cherbuin N, Anstey KJ. Development of the motivation to change lifestyle and health behaviours for dementia risk reduction scale. Dement Geriatr Cogn Dis Extra. 2014;4(2):172-183.

49. Ware JE, Sherbourne CD. The MOS 36-item short-form health survey (SF-36). I. Conceptual framework and item selection. Med Care. 1992; 30(6):473-483.

50. Brazier J, Roberts J, Deverill M. The estimation of a preference-based measure of health from the SF-36. J Health Econ. 2002;21(2):271-292.

51. Gibson RS. Principles of Nutritional Assessment. 2nd ed. New York: Oxford University Press; 2005.

52. Schröder H, Fitó M, Estruch R, et al. A short screener is valid for assessing Mediterranean diet adherence among older Spanish men and women. J Nutr. 2011;141(6):1140-1145.

53. Collins CE, Burrows TL, Rollo ME, et al. The comparative validity and reproducibility of a diet quality index for adults: the Australian Recommended Food Score. Nutrients. 2015;7(2):785-798.

54. National Health and Medical Research Council. Australian Dietary Guidleines. Available from: https://www.nhmrc.gov.au/guidelinespublications/n55. Accessed on 10 May 2018. 2013.

55. National Health and Medical Research Council. Australian Guide to Healthy Eating. Canberra: National Health and Medical Research Council; 2003.

56. Borg G. Borg's Perceived Exertion and Pain Scales. Champaign, IL: Human kinetics; 1998.
Clinical Interventions in Aging

\section{Publish your work in this journal}

Clinical Interventions in Aging is an international, peer-reviewed journal focusing on evidence-based reports on the value or lack thereof of treatments intended to prevent or delay the onset of maladaptive correlates of aging in human beings. This journal is indexed on PubMed Central, MedLine,

\section{Dovepress}

CAS, Scopus and the Elsevier Bibliographic databases. The manuscript management system is completely online and includes a very quick and fair peer-review system, which is all easy to use. Visit http://www.dovepress. com/testimonials.php to read real quotes from published authors. 